(1)

CrossMark

\title{
Can animal models really teach us anything about pneumonia? Con
}

\author{
Mark Metersky ${ }^{1}$ and Grant Waterer ${ }^{2}$
}

Affiliations: ${ }^{1}$ Dept of Medicine, Division of Pulmonary, Critical Care and Sleep Medicine, University of Connecticut School of Medicine, Farmington, CT, USA. ${ }^{2}$ Dept of Medicine, School of Medicine and Pharmacology and Lung Institute of Western Australia, University of Western Australia, Royal Perth Hospital, Perth, Australia.

Correspondence: Grant Waterer, University of Western Australia, School of Medicine and Pharmacology and Lung Institute of Western Australia, Level 4 MRF Building, Royal Perth Hospital, GPO Box X2213, Perth, Western Australia, 6000, Australia. E-mail: grant.watererQuwa.edu.au

@ERSpublications

Animal models of pneumonia are severely flawed and need to change if they are going to help us with new therapies http://bit.ly/2B1d7tD

Cite this article as: Metersky M, Waterer G. Can animal models really teach us anything about pneumonia? Con. Eur Respir J 2020; 55: 1901525 [https://doi.org/10.1183/13993003.01525-2019].

\section{Introduction}

In their 1987 community-acquired pneumonia (CAP) guidelines, the British Thoracic Society recommended amoxicillin with or without erythromycin (or tetracycline) in all admitted patients, with the coverage for Legionella being mandatory in seriously ill patients [1]. They also recommended intravenous flucloxacillin when Staphylococcus aureus was suspected and gentamicin or ceftazidime if a Gram-negative agent was suspected. This guideline, now more than 30 years old, also emphasised the need to identify critically ill patients based on objective physiological criteria so they could receive intensive care support, recommended obtaining culture specimens when possible and stated that antibiotics should be started immediately upon diagnosis. All of these 1987 recommendations were based on clinical studies on the aetiology of pneumonia, analysis of the predictors of outcome from pneumonia from clinical studies and a series of observational studies comparing outcomes of different antibiotic regimens in the prior three decades.

Fast forward 30 years and what has changed? Increasing penicillin resistance in pneumococci has driven a shift towards third-generation cephalosporins and/or respiratory fluroquinolones. Newer macrolides have replaced erythromycin. In some hospitals a blurring of hospital-acquired and community-acquired pathogens due to an increased frequency of comorbidities, frequent hospital contact and more widespread use of immunosuppressants has increased the need to consider covering pathogens like Pseudomonas aeruginosa and methicillin-resistant $S$. aureus. We have better validated severity tools for assisting clinicians in identifying patients who can be safely treated as outpatients, or who might need higher levels of inpatient care, such as in an intensive care unit. We have some debate over the utility of new tools like biomarkers and rapid diagnostic platforms, as well as the role of "protective" agents, like steroids, aspirin and statins, in subsets of patients and, especially, in those with severe disease [2].

Without exception, all of the changes since 1987 have come from insights generated by clinical studies, not laboratory experiments on animals. That is not to say there have not been improvements in many areas derived from laboratory science that are relevant to pneumonia, for example the marked reduction in pneumococcal disease with conjugate pneumococcal vaccines [3]; however, studies of animal pneumonia have not translated into any viable clinical treatment. 
It is also important to note that the lack of concordance between animal models and human disease is not without cost. Enormous amounts of money, time and effort have been devoted to large clinical trials of non-antimicrobial ancillary treatments for pneumonia and sepsis (of which up to 50\% was caused by pneumonia), with animal studies usually providing some of the evidentiary basis for these trials. Among the many examples are filgrastim (G-CSF) [4], anti-tumour necrosis factor antibodies [5] and anti-endotoxin antibodies [6]. Without exception, all of these treatments have ultimately been found to be ineffective or harmful in humans. Had it not been for the animal studies, perhaps the considerable resources and effort could have been applied to more relevant human studies.

There is no doubt that there is a need for better therapies for pneumonia. CAP remains a major health problem, ranking in the top six causes of death worldwide [7]. Equally, there is evidence that even with best possible practice there is little at this time that we can do to decrease mortality in patients with CAP [8]. Hospital-acquired and especially ventilator-associated pneumonia are major causes of morbidity, mortality and breeding grounds for antibiotic-resistant bacteria [9]. In defending the "con" argument of this pro/con debate, here we outline major problems with research into pneumonia using animal models, with the goal of redirecting efforts away from fundamentally flawed approaches to developing models with greater chance of delivering better therapeutic tools that clinicians and their patients need.

\section{Is the animal model relevant to human pneumonia?}

There is no question that animal models of pneumonia have pathology that looks identical to human pneumonia. However, animals in captivity, unlike humans in the wild, do not spontaneously develop pneumonia, at least not at a rate that makes it feasible to study the disease. Pneumonia must therefore be induced in animals so it can be studied, which creates a fundamental problem in adequately mimicking human disease. Humans do not present to emergency departments having just aspirated $10^{9} \mathrm{cfu} \cdot \mathrm{mL}^{-1}$ of pneumococci via syringe, yet this is the clinical scenario for animals acquiring pneumonia in the laboratory. The usual duration of symptoms in humans prior to presentation with pneumonia is days, and it is reasonable to hypothesise that infection was present for some time prior to the development of symptoms. In this "pre-pneumonia" time period, there are a large number of intrinsic, innate and adaptive immunological processes specifically designed to kill the invading pathogens and ameliorate damage from them. Indeed, it is known that in humans, the pattern of inflammatory response to pneumonia is in part dependent upon how long the patient was symptomatic prior to presentation [10].

The relative effectiveness of the host response compared to the virulence of the pathogen is a major factor in the enormous diversity of clinical presentations and outcomes from pneumonia. The host responses themselves are modified by additional factors such as genetics, epigenetics, comorbid illness and prior exposures, including vaccination and medications (including in many cases oral antibiotics). Despite the fact that mass aspiration of bacteria produces an identical pathological picture of pneumonia, given the large number of factors at play prior to patients presenting clinically with pneumonia, it is clear that immunologically and phenotypically the diseases must be different.

The impact of the initial size of the bolus of bacteria must also be considered a potential problem. While humans may develop acute lung injury from pneumonia, large inocula of bacteria may directly cause an acute toxic injury from bacterial antigens or bacterial toxins [11-14], rather than a progressive infective process, with obvious implications for assessment of immune response, pathogenesis and the therapeutic window. The characteristics of the inoculum in experimental pneumonia differs from human pneumonia in other ways. In humans, the inoculum usually is due to microaspiration of oropharyngeal secretions, which contains numerous species of microorganisms, many of which cause a unique pattern of inflammatory response $[15,16]$, even if only one of these organisms successfully evades host defence and results in pneumonia. To date very few pneumonia animal studies have employed anything other than a pure culture of a single organism [17]. Even the medium in which the bacteria are carried may play a role in the inflammatory response in human pneumonia. Aspiration of gastric secretions into the lung causes its own unique inflammatory pattern, at least in mice [18].

There is a clear need to develop alternative models of inducing pneumonia in animals. While convenient, a mass bolus of bacteria is far too limiting to understand human disease or how to successfully intervene to improve outcomes. Ideally any model would start with colonisation and progress to pneumonia over days, allowing time to study the interplay of evolving host responses that determine important clinical outcomes.

\section{The window of opportunity}

As outlined above, humans do not present prior to becoming infected asking for treatment for pneumonia they are about to have. In animal models however, pneumonia results from an intervention, so there is the possibility of giving experimental agents prior to exposure, at exposure and at intervals soon after 
exposure. With agents acting early in the pathophysiological development of pneumonia it is very unlikely that a window to intervene would still be open in the usual clinical situation. Therefore, any agent that does not show significant activity out to $48 \mathrm{~h}$ after exposure is very unlikely to have clinical utility, but despite this, most animal studies report interventions prior or at exposure or at most $6-8 \mathrm{~h}$ after. To be relevant to human disease, animal studies need to examine the impact of therapy beginning at least $24-$ $48 \mathrm{~h}$ post-exposure, given that this is typically at the lower end of the duration of symptoms in patients presenting to the hospital with pneumonia [19].

\section{Do mice respond the same way as man?}

Much more is known about the role of specific elements of the mouse inflammatory and immune responses through experimental manipulation than the human. While it is certainly true that many immunological responses are "ancient" and common to many species, for example Toll-like receptors [20], how clear is it that mouse response correlates to the human response?

A substantial body of work has compared immune pathways in mice and man and it is clear that, while there are significant commonalities, there are also marked differences [21-23]. These differences are more than sufficient to cast doubt on extrapolation to the human response, at least in the level of detail to adequately predict response to therapeutic interventions.

A further additional problem with laboratory animals is that they are deliberately kept in extremely clean environments without exposure to disease. Humans, however, contract a variety of acute and chronic infections throughout their lives, which almost certainly creates a very different immunological background compared to an infection-naïve animal [24], especially as there is evidence that wild mice and laboratory mice have very different immune responses to infection $[25,26]$. This is probably the most important dilemma for animal pneumonia researchers: their desire to control as many experimental variables as possible ultimately limits their ability to translate findings into what is a very complex human scenario.

\section{How relevant are animal outcomes to human outcomes?}

Inflammation, histological findings and mortality are generally the outcomes assessed in pneumonia animal studies. There is no doubt that mortality is an important outcome in human pneumonia, but well over $95 \%$ of human patients survive, and in these patients there are many important outcomes that are not assessed in animals. These include time to clinical stability, which is highly correlated with hospital length of stay. Time until return of function and time until return to work are extremely important outcomes to most patients recovering from pneumonia, yet cannot be measured in animals. Furthermore, even mortality, as assessed in most animal studies, is incompletely studied. Approximately half of pneumonia mortality occurs after discharge from the hospital [27], while animal studies generally assess only short-term mortality. Much of both early and late mortality is not directly caused by the acute infection. While humans die of cardiac events, strokes, pulmonary embolism, etc. [28], after a bout of pneumonia, these are rare in mice.

\section{Which mouse or rat?}

Animals with deliberately created differences in immune response induced by gene deletion or overexpression will obviously behave differently from an immunological standpoint. However, different "normal" or wildtype animal laboratory strains may also respond differently to the same pathogen. Fischer 344 and Sprague-Dawley rats have very different susceptibility to pneumonia due to Listeria [29]. BALB/c and C57BL/6 wildtype mice have different susceptibility and immune responses to Pasteurella pneumonia [30]. There are significant differences in susceptibility to influenza amongst common laboratory mice strains [31]. Clearly, the selection of animal strain may have a large impact on the outcome of any experiment, further questioning translation into human disease.

\section{What about the general milleu of comorbidity and ageing?}

Pneumonia is greatly increased in patients with comorbidities and advancing age. In particular, heart failure, COPD, diabetes, renal insufficiency, chronic liver disease and prior stroke all greatly increase the risk of acquiring pneumonia. Not only do these factors predispose to acquiring pneumonia, all except COPD have also been shown to be associated with worse outcomes from pneumonia [32]. Providing suitable animal models for these conditions is challenging, particularly as none of the human diseases are binary, they have a large range of severity modified by treatment.

As most patients who die from pneumonia are elderly, the large amount of literature regarding immunosenescence [33-35] has particular relevance in any consideration of animal models, where typically young or young-adult mice used. Attempts have been made to use elderly mice in pneumonia experiments [36-38], but how accurately they parallel human age-related change is unclear. Equally aged mice do not 
have the same level of chronic comorbid diseases as humans due to their strictly controlled diets and environments, further questioning how relevant aged mice are as an appropriate model for human elderly.

\section{Are larger animal models better than rodent models?}

While most of our argument has been focused on mice and rat models, in part because of the very large volume of research with rodents, larger animal models for pneumonia have been developed, including pigs, dogs, sheep and monkeys. While monkey models in particular may more closely replicate human immune responses, all these models still suffer from the basic problem of requiring a high initial inoculum to cause pneumonia. Large animal models are also expensive, especially monkeys, limiting the number of animals that can be studied, to the point that demonstrating reproducibility of results is an additional problem. Limitations due to comorbidity and ageing are also equally applicable in large animal models, as are problems with using an array of human pathogens. Therefore, while large animal models have some advantages, we still do not consider that there are any valid models to replicate human disease.

\section{What about the pathogen?}

Most human pathogens are not pathogenic for mice or indeed other animals under non-experimental conditions. This creates a further problem in translating human disease, if the pathogens have to be modified so they can infect and cause injury to the animal model.

For example, Streptococcus pneumoniae virulence is highly dependent on its polysaccharide capsule, of which more than 90 serotypes have been identified. More invasive serotypes (e.g. 1, 7 and 14) are more likely to cause infection when acquired and are much less likely to be found in the nose [39]. Less invasive serotypes (e.g. $9 \mathrm{~N}, 12 \mathrm{~F}, 22 \mathrm{~F}$ and $23 \mathrm{~A}$ ) are more likely to persist and spread, reaching a greater number of vulnerable hosts who may develop disease despite the lower virulence of the pathogen [40]. Incorporating these very important pathogen factors into an animal model is difficult.

Potentially even more important from a pathogen perspective is that there is increasing evidence that in many, if not the majority, of cases of human pneumonia two or more pathogens can be detected if a full array of molecular tests are employed $[19,41]$. Whether these represent concurrent infection, sequential infection, or more likely a mixture of both, remains unclear. However, at face value human pneumococcal pneumonia, up to $50 \%$ of which is associated with a co-pathogen, presents a very different scenario to a laboratory animal aspirating a large bolus of pneumococci.

It is clear that there are significant pathogen-driven differences that can be detected in the pathology of pneumonia produced in animal models [42]. While it does not necessarily follow that an intervention may be pathogen-specific, given that even pneumococcus accounts for probably less than $25 \%$ of all CAP [19, 41], demonstration of effect across multiple pathogens (including viruses) in any animal model would be required before having confidence in having developed a potentially useful human therapy.

\section{Conclusion}

We do not dispute that there has been significant new knowledge generated by studies of animal models of pneumonia. What is clear is that animal pneumonia in a laboratory is a tightly controlled, narrow focused, unidimensional disease that bears little resemblance to the very complex, messy and frequently multi-pathogen disease that is human pneumonia. It is therefore hardly surprising that we have no new therapies for human pneumonia that have arisen from animal model insights. Funding agencies, which often preferentially fund mechanistic studies as opposed to more clinically relevant studies, must accept some responsibility for this lack of progress. Instead of insisting on mechanistic studies, funders should support more clinical trials based on sound clinical and translational observations as these may provide more insights applicable to human pneumonia and treatment.

If basic science using animals to understand human pneumonia is to continue, then there must be changes to the experimental models we have described. Moving forward, scientists will need to embrace the complexity in terms of immunological background (i.e. prior exposures), age and comorbidity factors critical to driving outcomes from human disease, as persistence with existing paradigms is likely to continue to deliver what it has over the past three decades.

Conflict of interest: None declared.

\section{References}

1 Harrison BD, Farr BM, Connolly CK, et al. The hospital management of community-acquired pneumonia. Recommendations of the British Thoracic Society. J R Coll Physicians Lond 1987; 21: 267-269.

2 Wunderink RG, Waterer GW. Clinical practice. Community-acquired pneumonia. N Engl J Med 2014; 370: 543-551. 
3 Richter SS, Diekema DJ, Heilmann KP, et al. Changes in pneumococcal serotypes and antimicrobial resistance after introduction of the 13-valent conjugate vaccine in the United States. Antimicrob Agents Chemother 2014; 58: 6484-6489.

4 Root RK, Lodato RF, Patrick W, et al. Multicenter, double-blind, placebo-controlled study of the use of filgrastim in patients hospitalized with pneumonia and severe sepsis. Crit Care Med 2003; 31: 367-373.

$5 \mathrm{Li} \mathrm{XY}$, Donaldson K, Brown D, et al. The role of tumor necrosis factor in increased airspace epithelial permeability in acute lung inflammation. Am J Respir Cell Mol Biol 1995; 13: 185-195.

6 Burd RS, Cody CS, Raymond CS, et al. Anti-endotoxin monoclonal antibodies protect by enhancing bacterial and endotoxin clearance. Arch Surg 1993; 128: 145-150.

7 World Health Organization. The Top 10 Causes of Death. 2016. www.who.int/mediacentre/factsheets/fs310/en/ Date last updated: 24 May 2018.

8 Waterer GW, Self WH, Courtney DM, et al. In-hospital deaths among adults with community-acquired pneumonia. Chest 2018; 154: 628-635.

9 Kalil AC, Metersky ML, Klompas M, et al. Management of adults with hospital-acquired and ventilator-associated pneumonia: 2016 clinical practice guidelines by the Infectious Diseases Society of America and the American Thoracic Society. Clin Infect Dis 2016; 63: e61-e111.

10 Mendez R, Menendez R, Cilloniz C, et al. Initial inflammatory profile in community-acquired pneumonia depends on time since onset of symptoms. Am J Respir Crit Care Med 2018; 198: 370-378.

11 Tasaka S, Kamata H, Miyamoto K, et al. Intratracheal synthetic CpG oligodeoxynucleotide causes acute lung injury with systemic inflammatory response. Respir Res 2009; 10: 84.

12 Malloy JL, Veldhuizen RA, Thibodeaux BA, et al. Pseudomonas aeruginosa protease IV degrades surfactant proteins and inhibits surfactant host defense and biophysical functions. Am J Physiol Lung Cell Mol Physiol 2005; 288: L409-L418.

13 Wiener-Kronish JP, Sakuma T, Kudoh I, et al. Alveolar epithelial injury and pleural empyema in acute $P$. aeruginosa pneumonia in anesthetized rabbits. J Appl Physiol 1993; 75: 1661-1669.

14 Chang SW, Czartolomna J, Voelkel NF. Role of eicosanoids in staphylococcal alpha-toxin-induced lung injury in the rat. Am J Physiol 1992; 262: 4 Pt. 1, L502-L510.

15 Burgmeijer EH, Duijkers R, Lutter R, et al. Plasma cytokine profile on admission related to aetiology in community acquired pneumonia. Clin Respir J 2019; 13: 605-613.

16 Zobel K, Martus P, Pletz MW, et al. Interleukin 6, lipopolysaccharide-binding protein and interleukin 10 in the prediction of risk and etiologic patterns in patients with community-acquired pneumonia: results from the German competence network CAPNETZ. BMC Pulm Med 2012; 12: 6.

17 Hraiech S, Papazian L, Rolain JM, et al. Animal models of polymicrobial pneumonia. Drug Des Devel Ther 2015; 9: 3279-3292.

18 Appel JZ 3rd, Lee SM, Hartwig MG, et al. Characterization of the innate immune response to chronic aspiration in a novel rodent model. Respir Res 2007; 8: 87.

19 Jain S, Self WH, Wunderink RG, et al. Community-acquired pneumonia requiring hospitalization among U. S. adults. N Engl J Med 2015; 373: 415-427.

20 Moresco EM, LaVine D, Beutler B. Toll-like receptors. Curr Biol 2011; 21: R488-R493.

21 Denas O, Sandstrom R, Cheng Y, et al. Genome-wide comparative analysis reveals human-mouse regulatory landscape and evolution. BMC Genomics 2015; 16: 87.

22 Seok J, Warren HS, Cuenca AG, et al. Genomic responses in mouse models poorly mimic human inflammatory diseases. Proc Natl Acad Sci USA 2013; 110: 3507-3512.

23 Shay T, Jojic V, Zuk O, et al. Conservation and divergence in the transcriptional programs of the human and mouse immune systems. Proc Natl Acad Sci USA 2013; 110: 2946-2951.

24 Tao L, Reese TA. Making mouse models that reflect human immune responses. Trends Immunol 2017; 38: $181-193$

25 Reese TA, Bi K, Kambal A, et al. Sequential infection with common pathogens promotes human-like immune gene expression and altered vaccine response. Cell Host Microbe 2016; 19: 713-719.

26 Beura LK, Hamilton SE, Bi K, et al. Normalizing the environment recapitulates adult human immune traits in laboratory mice. Nature 2016; 532: 512-516.

27 Metersky ML, Waterer G, Nsa W, et al. Predictors of in-hospital vs postdischarge mortality in pneumonia. Chest 2012; 142: 476-481.

28 Mortensen EM, Coley CM, Singer DE, et al. Causes of death for patients with community-acquired pneumonia: results from the Pneumonia Patient Outcomes Research Team cohort study. Arch Intern Med 2002; 162: 1059-1064.

29 Antonini JM, Roberts JR, Clarke RW. Strain-related differences of nonspecific respiratory defense mechanisms in rats using a pulmonary infectivity model. Inhal Toxicol 2001; 13: 85-102.

30 Fornefett J, Krause J, Klose $\mathrm{K}$, et al. Comparative analysis of humoral immune responses and pathologies of $\mathrm{BALB} / \mathrm{c}$ and C57BL/6 wildtype mice experimentally infected with a highly virulent Rodentibacter pneumotropicus (Pasteurella pneumotropica) strain. BMC Microbiol 2018; 18: 45.

31 Leist SR, Pilzner C, van den Brand JM, et al. Influenza H3N2 infection of the collaborative cross founder strains reveals highly divergent host responses and identifies a unique phenotype in CAST/EiJ mice. BMC genomics 2016 17: 143.

32 Mandell LA, Wunderink RG, Anzueto A, et al. Infectious Diseases Society of America/American Thoracic Society consensus guidelines on the management of community-acquired pneumonia in adults. Clin Infect Dis 2007; 44: Suppl. 2, S27-S72.

33 Fukushima Y, Minato N, Hattori M. The impact of senescence-associated T cells on immunosenescence and age-related disorders. Inflamm Regen 2018; 38: 24.

34 Drew W, Wilson DV, Sapey E. Inflammation and neutrophil immunosenescence in health and disease: targeted treatments to improve clinical outcomes in the elderly. Exp Gerontol 2018; 105: 70-77.

35 Goncalves MT, Mitchell TJ, Lord JM. Immune ageing and susceptibility to Streptococcus pneumoniae. Biogerontology 2016; 17: 449-465. 
36 Janesch P, Stulik L, Rouha H, et al. Age-related changes in the levels and kinetics of pulmonary cytokine and chemokine responses to Streptococcus pneumoniae in mouse pneumonia models. Cytokine 2018; 111: 389-397.

37 Shrivastava P, Watkiss E, van Drunen Littel-van den Hurk S. The response of aged mice to primary infection and re-infection with pneumonia virus of mice depends on their genetic background. Immunobiology 2016; 221: 494-502.

38 Williams AE, Jose RJ, Brown JS, et al. Enhanced inflammation in aged mice following infection with Streptococcus pneumoniae is associated with decreased IL-10 and augmented chemokine production. Am J Physiol Lung Cell Mol Physiol 2015; 308: L539-L549.

39 Brueggemann AB, Griffiths DT, Meats E, et al. Clonal relationships between invasive and carriage Streptococcus pneumoniae and serotype- and clone-specific differences in invasive disease potential. J Infect Dis 2003; 187: 1424-1432.

40 Fenoll A, Ardanuy C, Linares J, et al. Serotypes and genotypes of $S$. pneumoniae isolates from adult invasive disease in Spain: a 5-year prospective surveillance after pediatric PCV13 licensure. The ODIN study. Vaccine 2018; 36: 7993-8000.

41 Holter JC, Muller F, Bjorang O, et al. Etiology of community-acquired pneumonia and diagnostic yields of microbiological methods: a 3-year prospective study in Norway. BMC Infect Dis 2015; 15: 64.

42 Dietert K, Gutbier B, Wienhold SM, et al. Spectrum of pathogen- and model-specific histopathologies in mouse models of acute pneumonia. PLoS One 2017; 12: e0188251. 